\title{
Initial Experience With Automatic Image Transmission to an Intensive Care Unit Using Picture Archiving and Communications System Technology
}

\author{
Carl E. Ravin
}

\begin{abstract}
This report describes a refinement of the interaction between a Computed Radiography (CR) system and a Picture Archiving and Communications System (PACS) that has made possible automatic distribution of images to specified locations. Those interactions between the CR and the PACS that were considered important to the acceptance of the data to be sent to the intensive care unit (ICU) are described. Display and user interface considerations important for physician acceptance of the unit are also discussed. System enhancements and future applications will be developed based on the possible implications of this technology in both Radiology and the ICUs.

(C) 1990 by Society of Photo-optical Instrumentation Engineers.
\end{abstract}

KEY WORDS: PACS, computed radiography, automatic transmission, intensive care unit, utilization. softcopy viewing.

A MAJOR ADVANTAGE of digital imaging is the potential ability to directly transmit images to locations throughout the hospital. Previously we have reported on the success of a full time commercial interface between a computed radiography (CR) system and a Picture Archiving and Communications System (PACS) network. ${ }^{1}$ This integrated system provides the minimum capability necessary for the establishment of an all-digital radiology department. For the first time it provides a full time, all digital, commercial quality interface for PACS acquisition, storage, and display of exams traditionally performed in conventional radiology areas.

The first application of this integrated system has been implemented at Duke University (Durham, NC) as a means of sending images to an intensive care unit (ICU) as a replacement both for sending copies of film to the unit and having the physician visit the radiology department to view film. It has been in operation in an initially semiautomatic and now fully automatic mode since July, 1989. Over 2000 images have been transmitted to the Coronary Care Unit (CCU) using this system. Over 1700 new exams consisting of one or more images have been viewed in the CCU. In addition, previous exams, when available, are displayed automatically for comparison. Usage of the workstation has been constant since it was introduced, indicating a degree of acceptance and even enthusiasm for the system and the technology.

\section{INTENSIVE CARE UNIT ENVIRONMENT AND RELATION TO RADIOLOGY}

It is highly desirable for the physicans in ICUs to have immediate and complete access to the radiology data they have requested if they are to care for their patients properly. This data could be in the form of conversations, formal and informal reports, as well as the raw radiographic data. Until recently, the only way raw radiographic data could be provided was by having copies of radiographs made and delivered to the unit, or by having the ICU physician come to the radiology department to review the films with or without the benefit of a radiologist for consultation. The ICU physician usually needs information quickly to determine the best course of treatment for critically ill patients. In others, the need to know exists, but access to the data need not be immediate (short-interval). Nevertheless, if having a copy (hard or soft) saves the physician a trip to radiology, the physician is given a little more time to handle the most pressing cases. Several physicians can discuss cases throughout the day and night when the images and other radiology data are made available in the CCU. This makes local availability of image data the best choice in cases where both immediate and short-interval care are required.

The interaction between the radiologist and ICU physicians has always been an important relationship. The introduction of new technology and different procedures can be stressful to all

From the Radiology Department, Duke University Medical Center, Durham, NC.

Address reprint requests to Carl E. Ravin, MD, Department of Radiology, Box 3808, Duke University Medical Center, Durham, NC 27710.

(c) 1990 by Society of Photo-optical Instrumentation Engineers.

Figures 1-4@ 1990 by W.B. Saunders Company. 0897-1889/90/0303-0004\$03.00/0 
parties involved. Changes that will effect the way a bedside physician deals with a radiologist must be handled in a way that will not interfere with the overall goal of improving patient care. The combination of reference materials-reports, copies of film, verbal consultations, etc - along with daily conferences have made the interaction between the radiologist and the bedside physician a strong one.

\section{SOFTCOPY VIEWING IN AN INTENSIVE CARE UNIT}

A significant change in the traditional method of operation began at Duke University in 1988 when the radiology department began using computed radiography (CR) for some of the portables done in the ICUs. The Philips Computed Radiography (PCR) system (Shelton, CT) gave the units a needed improvement: consistent image quality. Variations because of differing radiographic techniques and technologists were effectively eliminated. After a short time, the benefits were clear to both the radiologist and the ICU physicians. Technologists also greatly appreciated the technology because of the ease with which exposures could be made. In cases where copies of an exam were needed to send to the ICU, two copies of the original were generated, and thus, the "copy" was the equivalent of the original in every detail.

When the PCR was interfaced to PACS (the CommView system, AT\&T and Philips), the possibility of viewing images on monitors rather than by using film became a reality. This integrated system has provided the backbone upon which several important and interesting applications may be built. One of these applications for the integrated system at Duke University lay in the area of transmitting images to an ICU where there remains a need for fast access to exam results.

As part of the controlled introduction and evaluation of the ICU application package- $-\mathrm{CR}$, PACS, and ICU transmissions-the radiology department continued to use hard copy for primary diagnosis, but one ICU was given a Critical Care Workstation (CCW) for use instead of copies of film. Daily conferences in the radiology department among the ICU physicians and the radiologists continued as usual. The primary effect of the addition of the CCW was expected to be (1) in those cases where changes in the patient condition occurred after the morning conference; (2) when new patients were admitted during the day; (3) late in the evening when it might be inconvenient to come to radiology to view film; or (4) when a radiologist was not available to do an immediate interpretation.

The CCW was to be used in those consultative environments where copy film or no film would have been used on the unit previously. It was designed to provide ICU physicians with the ability to review important exams locally and make them available almost as quickly as they would be to a radiologist in radiology. Local image storage and viewing allow the referring physicians to manage their time better.

\section{Design and Use of the Critical Care Workstation}

Before any system was introduced into the chosen ICU, it had to meet certain operational criteria. First, the image quality had to be such that the physicians viewing the images would be able to discern those features they tended to be most interested in seeing: tube and catheter placements, extensive pulmonary opacities, and potential complications such as lobar collapse or pneumothorax. This was particularly true during those times when a radiologist was not readily available and when immediate assessments of tube placements was important.

Image presentation was a significant consideration. In most of the cases transmitted, the received data had to be in a form requiring the least amount of user interaction. The proper image orientation had to be presented most of the time and window-level values had to be optimal. Comparisons of new and older films was considered a necessity, making the use of two screens for viewing preferable. Minimum interaction with the system by the users was felt to be crucial. It was not considered acceptable to have the normal users involved in any activity that would require a significant wait or prolonged interaction, forcing the system operation and the user interface to be critical issues in the effective implementation of the system. Several issues were important in the design of the user interface. One of the most important of these was the 
desire to make the interface as easy to use as possible, while at the same time giving the users the flexibility necessary to do their job well. The primary goal in designing the system was making it a tool that could be used regularly and simply. After several meetings, the present interface was developed and is now being used in the CCU. A key feature of this interface is that it is entirely mouse driven. The physician merely has to select the patient desired from a patient work list and the images are automatically displayed. Additional images and image manipulation featureswindow-level, image orientation and zoom-are available at any time, also under mouse control.

The unit in the CCU is equipped with two landscape mode monitors, each with a maximum $1280 \times 1024$ matrix. The CPU is equipped with a 300 Mbyte disk drive for local storage of acquired images. The connection to the rest of the network is made via Ethernet.

To minimize interaction of the final users with the system, the images were transmitted automatically to the unit, and the names and type of the most recent exam presented to give the user an indication at a glance of the status of the patient's most recent exam. Consequently, the physician was able to determine availability of an exam with little or no interaction with the unit.

The information needed to direct the acquired images to the appropriate workstation was passed to the PACS through the interface with the PCR. Based on the information sent to the CommView system, the acquired image was then routed to the appropriate workstation(s). Image data could be sent to any number of workstations, based on configuration parameters set up in the PACS.

Over the 7 months the system has been in operation, its use as a tool for reviewing acquired radiographic images in the $\mathrm{CCU}$ has been good. To quantify the usage and usage patterns of the $\mathrm{CCW}$, a log function was added to record patient identification and the date and time of each use. Over the 6 months (August through January) that usage has been automatically recorded, over 1700 exams have been selected from the patient list, with an average of almost 11 exams per day. This compares with over 2000 exams transmitted to the CCU in the same period of time.

A significant number of the exams selected were selected more than once during the day, indicating an exam of particular interest. This type of usage suggests the daily conference in the radiology department is particularly effective in informing the CCU intensivists of the patient's condition after the morning rounds. The intensivists seem to prefer receiving images on the $\mathrm{CCW}$ over receiving copies of film, and have several times called to have images sent up to the unit when, for one reason or another, the images were not transmitted initially. Figures 1, 2, and 3 show the average daily utilization of the system by day, month, and hour. Over the 6 months of availability, the average daily utilization shows a smooth variation, ranging from a maximum of about 12 uses per day down to about 9 uses per day during December. This drop is attributed to changes in association with the Christmas holidays. The average utilization returned to the usual levels in January. When viewed as a function of the days of the week, the utilization indicates a preference to view images certain days of the week, with Monday as the day the system was used most. Significantly more usage on Friday, Sunday and Monday counterbalance the less then average use during the middle of the week and on Saturday. As a function of time, afternoon and evening hours seem to be the times preferred. The nearly level hourly distribution suggests that there is no pattern to be disclosed. A significant contributor to the hourly viewing distribution was the continuance of the morning conference in the radiology department after the morning round films were completed.

Figure 4 shows the number of times a particular patient was selected for viewing once during a day and more than once, with the maximum

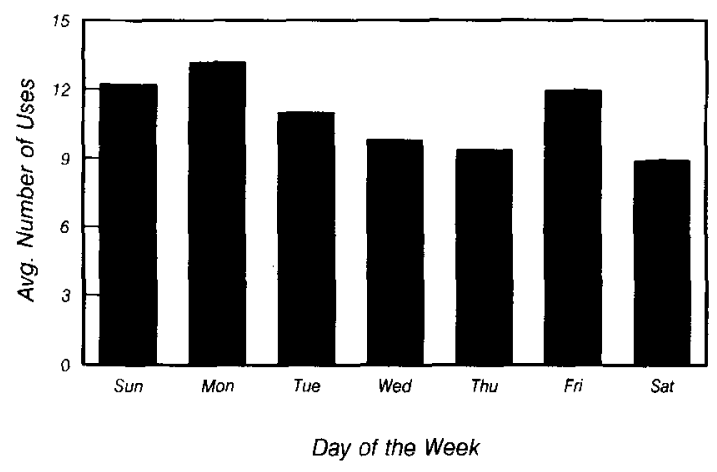

Fig 1. Average daily utilization by day. 


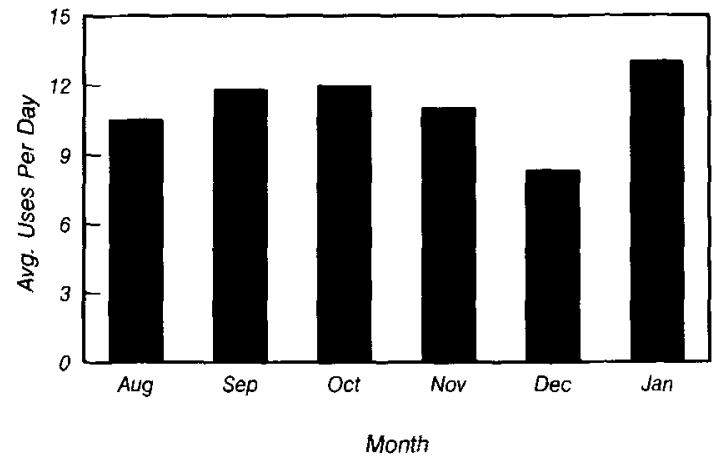

Fig 2. Average daily utilization by month.

number of recorded selections being 13 in a single day. The average daily census of the unit over that same period of time was constant at between 13 and 14 patients per day in a unit with 16 intensive care beds.

\section{DISCUSSION}

\section{Benefits, Pitfalls, and Shortcomings}

The benefits of the total system have been obvious. The computed radiography PCR system allows for very consistent examinations in a mobile radiography environment. This greatly facilitates comparison of one examination to another and has been extremely well received by the cardiologists in the ICU. In addition, the automated transmission of images occurs in a time frame very similar to that required to process the data stream into a film. In other words, the image is available in the CCU at the same time that a film is produced in the radiology department. As the ICU is separated from the radiology department by approximately 15 to 20 minutes of travel time, the savings to individuals in terms of time by not necessarily having to

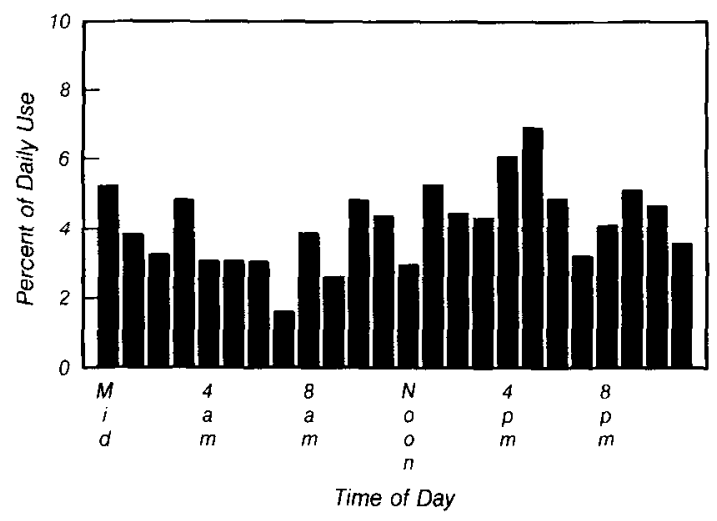

Fig 3. Percent utilization by hour.

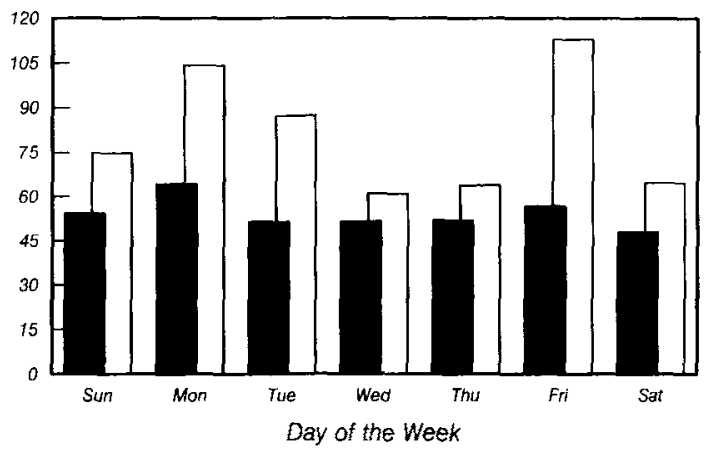

Two or More $\square$ Single Selection

Fig 4. Single versus multiple selection by day of the week.

come to the radiology department is very significant.

Utilization of the system has demonstrated that, somewhat unexpectedly, occasional patients remain in the unit for prolonged periods. Currently, we have one patient who has been in the unit for more than 90 days and another currently approaching 60 days. Given the number of radiographic examinations these patients undergo, it is possible that, by themselves, they could occupy a significant proportion of local disk storage. Thus it has been necessary to purge examination records from individual patient files as well as to purge patient records from the local disk when they are transferred out of the unit. This requires oversight by the department of radiology which might be viewed as a shortcoming, although it has proven to be of minimal inconvenience.

A potential pitfall for the radiology department is that the ability to view the images remotely in the ICU, with all of its inherent convenience, could ultimately mean that there would be less desire for face to face conferences between radiologists and intensivists. Interestingly, this has not yet occurred, in large part because the intensivists recognize that there are subtleties on the radiographic examinations that are more apparent to radiologists, and therefore, they wish to continue the interactions. It should be noted, however, that our system was introduced after a face to face daily conference has been in place for many years. Thus, the tradition is the morning conference. In other environments where such a conference does not exist, it is possible that the convenience of direct remote 
transmission to the ICU might result in decreased interactions between radiologists and intensivists.

One overriding benefit of the system is its ability to get radiology information out of the department to the physicians that use it to treat their patients. However, this same feature could also viewed as a pitfall. Significant numbers of radiologists and hospital personnel at other institutions believe that radiographic data should not leave the radiology department without a completed report to accompany it. To do otherwise could introduce the risk of harming radiology relations with the unit by allowing uninterpreted radiology-produced information to go to the units. In those cases, several other operations need to be well coordinated for the application to be beneficial. A delay of several hours between the time data was acquired and the time the images were sent to the unit could result in a completely different type of application, and a much narrower scope, both real and perceived. A report, in some form, would have to be available to send with the image within moments after the interpretation has been completed. And the interpretation would have to be done within moments after the exam was completed by the technologist.

During the time images have been sent directly to the unit, no detrimental effects on the relation- ship between the radiology department and the ICU physicians have been observed because of the lack of reports. Completed reports are desired for all cases in the unit, but their absence has not been an obstacle.

We expect that the use of the workstation in the ICUs, along with the use of a similar diagnostic workstation in radiology, will stimulate the interactions between the intensivists and the radiologists both during the conference and later by phone when additional images or complications make communication advisable.

\section{SUMMARY}

An effective system for automated direct transmission of ICU images to the local ICU has been implemented and has functioned successfully. Initial responses to the system have been most positive and other ICUs are currently anxious to be added to the network. This would seem to underscore the positive reception on the part of intensivists to systems of this sort. As we add ICUs, the problems of more examinations and more data to handle will be more fully explored.

\section{REFERENCES}

1. Ravin CE: Digital Radiography and PACS. Medical Imaging III: PACS System Design and Evaluation. SPIE Proc 1093:362-366, 1989 